\title{
Essais industriels \\ sur le comportement d'une turbine-pompe mono-étage de haute chute
}

\author{
Industrial tests on the behaviour of a single-stage pump-turbine operating \\ under high head
}

Paul Jarriand, Jean Lathuile et Gilbert Caillot

Société Neyrpic - Grenoble

\section{Généralités}

L'évolution des turbines-pompes monocellulaires vers des chutes toujours plus élevées nécessite un contrôle rigoureux sur prototype des hypothèses de calcul adoptées et la connaissance précise du comportement des différents organes de la machine dans tous les cas de fonctionnement permanent ou transitoire.

Nous nous proposons de présenter ici un certain nombre de résultats de mesures effectuées sur un des quatre groupes turbine-pompe équipant la centrale du Tajo de la Encantada (Espagne). Cet aménagement réalisé par la Compania Sevillana De Electricidad est situé sur le Rio Guadalhorce au nord de Malaga.

Le bassin inférieur est constitué par le lit de la rivière barré par une digue équipée d'un évacuateur de crues.

Le bassin supérieur est artificiel; il comporte une tranche utile de $2622 \mathrm{Hm}^{3}$ entre les cotes $566,5 \mathrm{~m}$ et $586,5 \mathrm{~m}$.

- Une galerie en charge de 5,4 m de diamètre et d'environ $520 \mathrm{~m}$ de longueur relie le bassin supérieur à la cheminée d'équilibre.

- Une conduite forcée de diamètre compris entre $5,4 \mathrm{~m}$ et $4,4 \mathrm{~m}$ sur $500 \mathrm{~m}$ de longueur, se subdivise en 2 rameaux de $3,4 \mathrm{~m}$ de diamètre et de $300 \mathrm{~m}$ de longueur alimentant chacun 2 groupes turbine-pompe de la centrale, située au bord de la retenue aval.

Les conduits basse pression se limitent donc aux aspirateurs des groupes. Ils sont équipés chacun d'une vanne plate.

Côté haute pression, les groupes sont protégés par un robinet sphérique.

\section{Caractéristiques des groupes}

Les quatre groupes turbine-pompe équipant la centrale du Tajo de la Encantada sont du type monoétage à distributeur mobile. Leur ligne d'arbre, à axe vertical, est guidée par trois paliers, le pivot est disposé sur le croisillon supérieur de l'alternateur.

Un moteur Poney permet le lancement du groupe en pompe après dénoyage de la roue de turbine.

Les caractéristiques de ces machines sont résumées ci-après :

\begin{tabular}{|l|c|c|}
\hline & Turbine & Pompe \\
\hline Niveau amont & $586,5 / 566,5$ & $586,5 / 566,5$ \\
Niveau aval & $202 / 188$ & $202 / 188$ \\
Chutes brutes (m) & $398,5 / 364$ & $398,5 / 364$ \\
Débit (m $3 / \mathrm{s}$ ) & $25,6 / 28,7$ & $22,35 / 26,15$ \\
Puissance (MW) & limitée à $92 \mathrm{MW}$ & 102 \\
& sous $378 \mathrm{~m}$ & \\
Vitesse de rotation & 500 & 500 \\
(tr/mn) & \multicolumn{2}{|c|}{49 à $51 \mathrm{~Hz}$} \\
Variation de fré- & \multicolumn{2}{|c|}{} \\
quence & \multicolumn{2}{|c|}{} \\
\hline
\end{tabular}

Les groupes doivent également fonctionner en compensateur synchrone en tournant dans le sens turbine.

Mesures effectuées sur une turbine-pompe

Les essais réalisés ont eu essentiellement pour objet de vérifier le comportement des organes de vannage, de la ligne d'arbre et des paliers ; d'observer l'évolution des pressions hydrauliques en différents points de la 


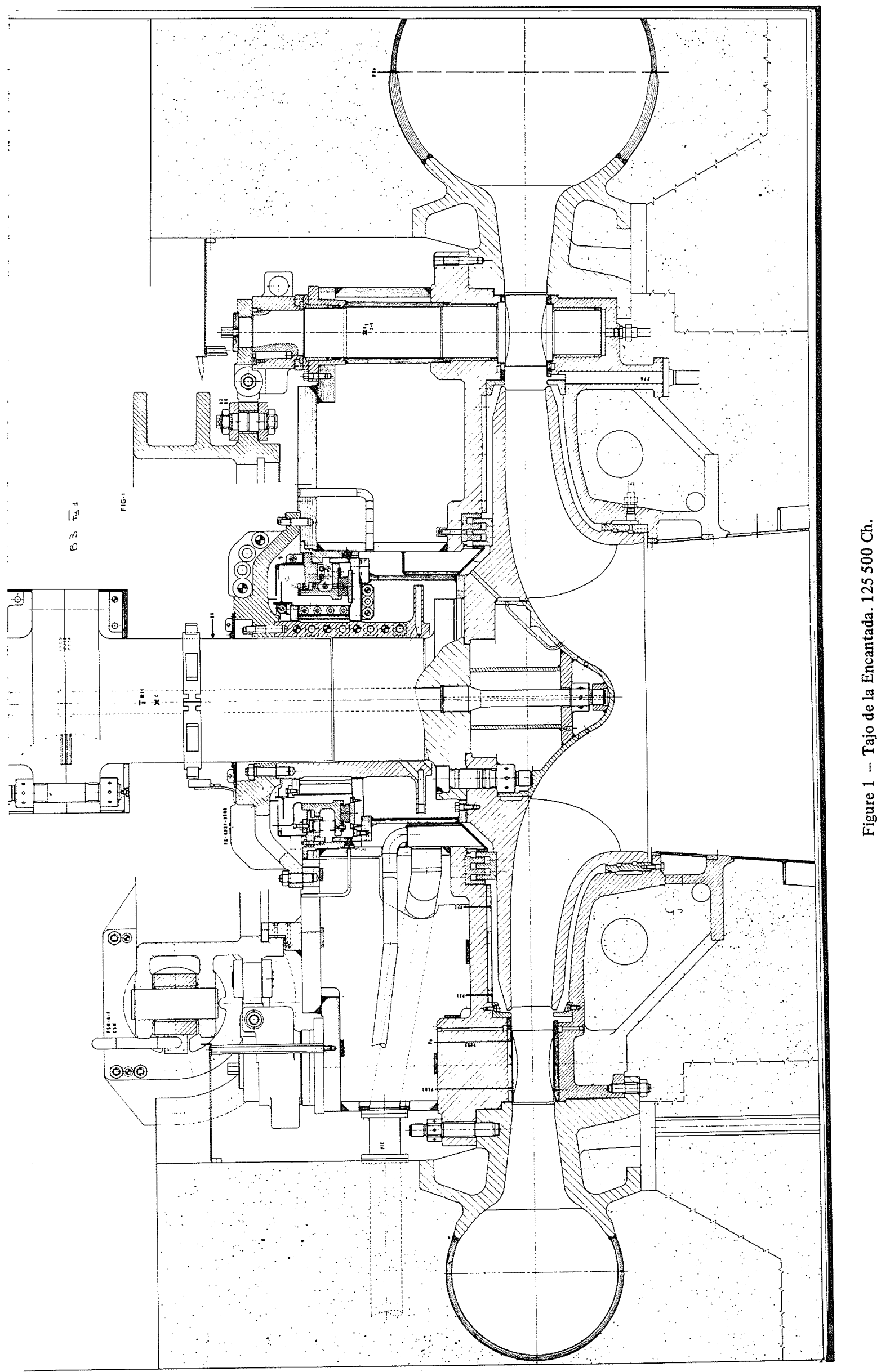


machine et ceci en régime permanent et en régime transitoire pour les différents modes de fonctionnement.

Au cours des essais, les paramètres suivants ont été enregistrés

- couple sur le tourillon de deux directrices

- couple de torsion et moment de flexion sollicitant l'arbre (ces signaux sont retransmis par télémesure)

- poussées radiales sur le palier turbine et sur les deux paliers alternateurs mesurées suivant deux directrices perpendiculaires

- poussée axiale

- pression dans la bâche

- pression en deux points du canal diffuseur (canal constitué par deux directrices consécutives, l'une d'elle étant équipée d'un pont de torsion pour la mesure du couple).

L'emplacement des points de mesures est précisé sur la figure 1 .

\section{Mesures sur le groupe à l'arrêt}

Avant la mise en service de la machine, différents essais ont permis de contrôler les valeurs théoriques des rigidités des paliers, des fréquences propres de la ligne d'arbre et des directrices, et d'étalonner les dispositifs de mesure des poussées radiale et axiale.

\section{Rigidité des paliers}

Pour cette mesure, l'arbre guidé par 3 paliers a été rendu isostatique en retirant alternativement les patins des paliers intermédiaire et supérieur. Un vérin hydraulique permettait d'appliquer une charge radiale croissante sur la ceinture de la roue d'une part, et sur le plateau d'accouplement d'autre part. Les déplacements des paliers étaient mesurés avec des comparateurs fixés sur la structure en béton de la centrale, les poussées étant exercées suivant deux directions perpendiculaires. Ces essais ont permis de contrôler l'isotropie des paliers et de confirmer les valeurs des flexibilités théoriques utilisées pour les calculs dynamiques de la ligne d'arbre.

\section{Fréquence propre de la flexion de la ligne d'arbre}

$\mathrm{Au}$ cours de cet essai, les patins des paliers supérieur et intermédiaire étaient fortement serrés contre l'arbre. Celui-ci était également immobilisé au niveau du palier turbine par un dispositif comportant 8 butons de blocage. Un excitateur constitué d'un vérin hydraulique permettait d'appliquer sur le plateau d'accouplement, une force sinusoïdale de $1250 \mathrm{dAN}$ de fréquence variable entre 2 et $30 \mathrm{~Hz}$.

La figure 2 représente la courbe de réponse de l'arbre au voisinage de sa première fréquence propre qui atteint 17.2 Hz. Le calcul avait donné une première fréquence propre de $17 \mathrm{~Hz}$.

Il n'a pas été possible de reprendre cet essai après mise en eau de la machine, ce qui aurait permis d'évaluer l'influence de la masse ajoutée et de l'amortissement dus à la présence du fluide baignant la roue.

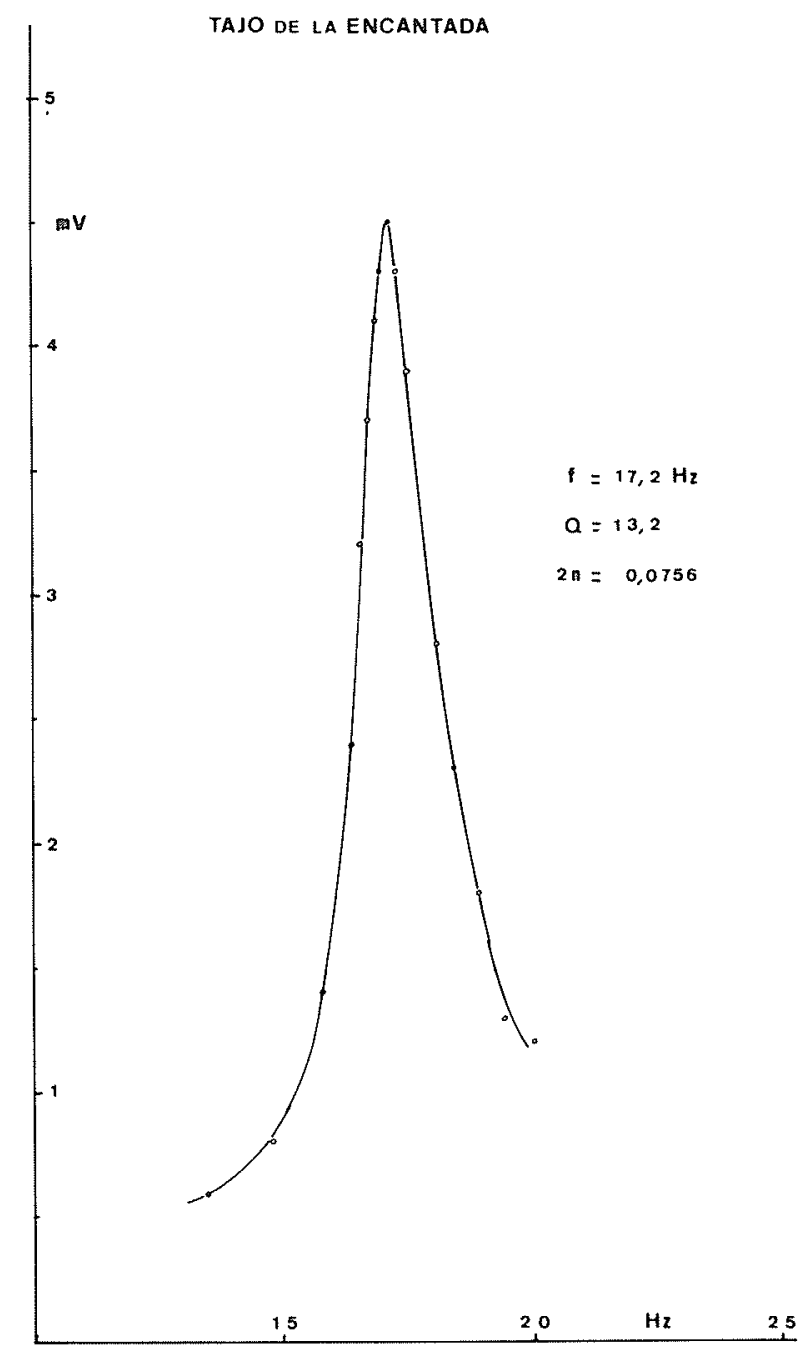

Figure 2 -

\section{Essai de vibrations des directrices}

La mesure de la fréquence propre dans l'air du système constitué par une directrice et son embiellage de commande a été réalisée en appliquant une force d'excitation sur le profil. L'essai a confirmé la valeur théorique.

\section{Fréquences caractéristiques}

Nous résumons ci-après quelques fréquences caractéristiques que nous retrouverons dans les enregistrements en cours des essais dynamiques.

- fréquence de rotation

$$
f_{0}=8,33 \mathrm{~Hz}
$$

- fréquence d'excitation due aux aubages de la roue

- fréquence d'excitation due aux sillages des directrices

$$
Z f_{0}=75 \mathrm{~Hz}
$$

- fréquence propre de l'arbre (flexion)

- fréquence propre de l'arbre (torsion) $=18,6 \mathrm{~Hz}$

- fréquence propre de torsion d'une directrice

$$
=260 \mathrm{~Hz}
$$




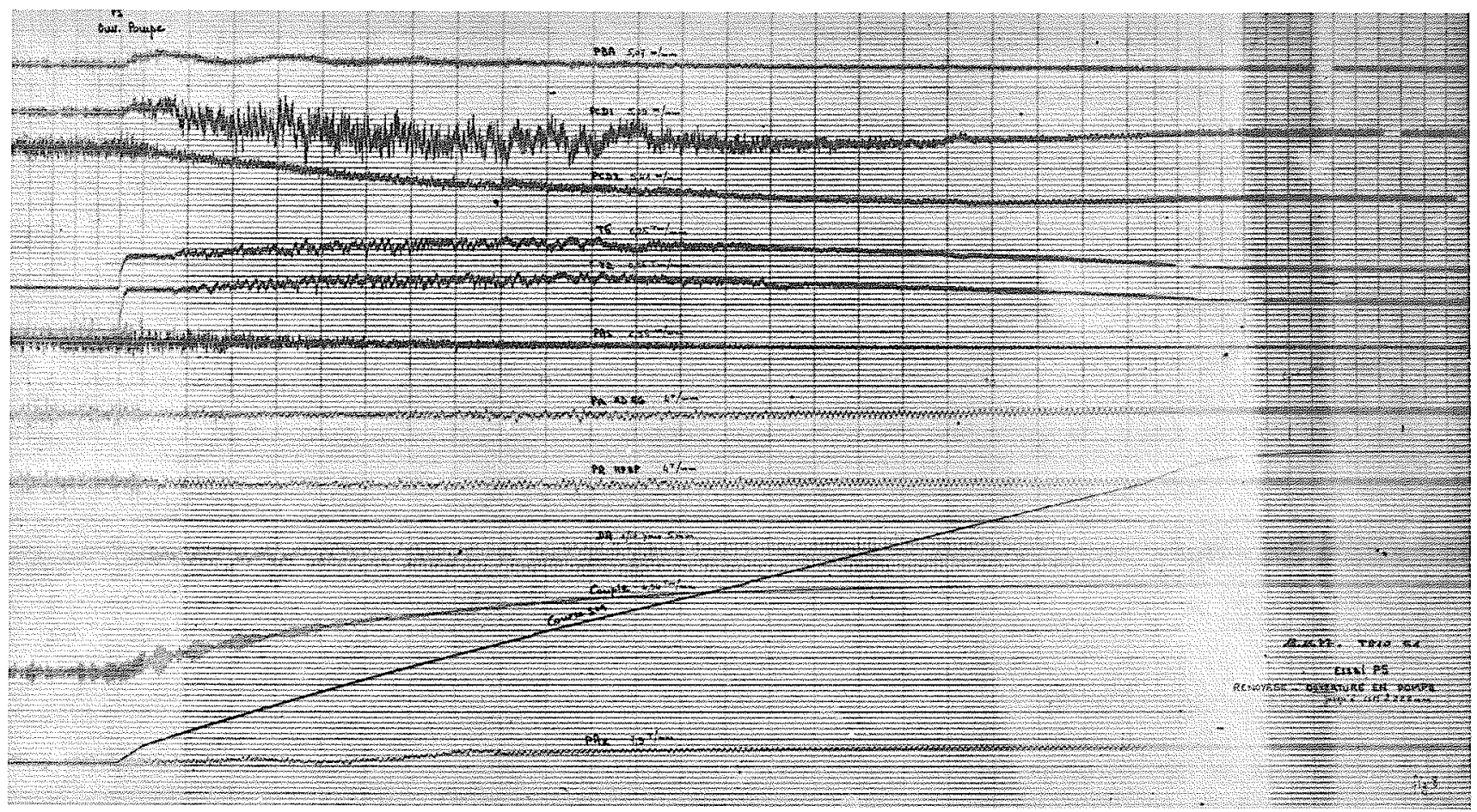

Figure 3 -

\section{Essais en rotation}

En raison de l'absence d'apports, le bassin supérieur était entièrement vide au moment de la mise en service du premier groupe.

Le démarrage de cette machine a donc eu lieu en pompe, les adductions haute pression ayant été au préalable remplies jusqu'au seuil de la vanne de tête. Le remplissage du bassin supérieur jusqu'à la cote minimale d'exploitation a été effectué avec une ouverture réduite du distributeur afin de limiter le débit.

\section{Démarrage du groupe en pompe}

Le lancement du groupe est assuré par un moteur Poney, la roue étant dénoyée et la bâche à pression réduite : $15 \mathrm{~m}$ d'eau au-dessus du niveau aval.

Après synchronisation et couplage du groupe, le renoyage de la pompe est réalisé en 60 secondes environ. Le remplissage s'effectue d'une part par l'amont (ouverture du robinet sphérique et remplissage de la roue jusqu'à l'œillard) et par l'aval : remontée du niveau en évacuant l'air.

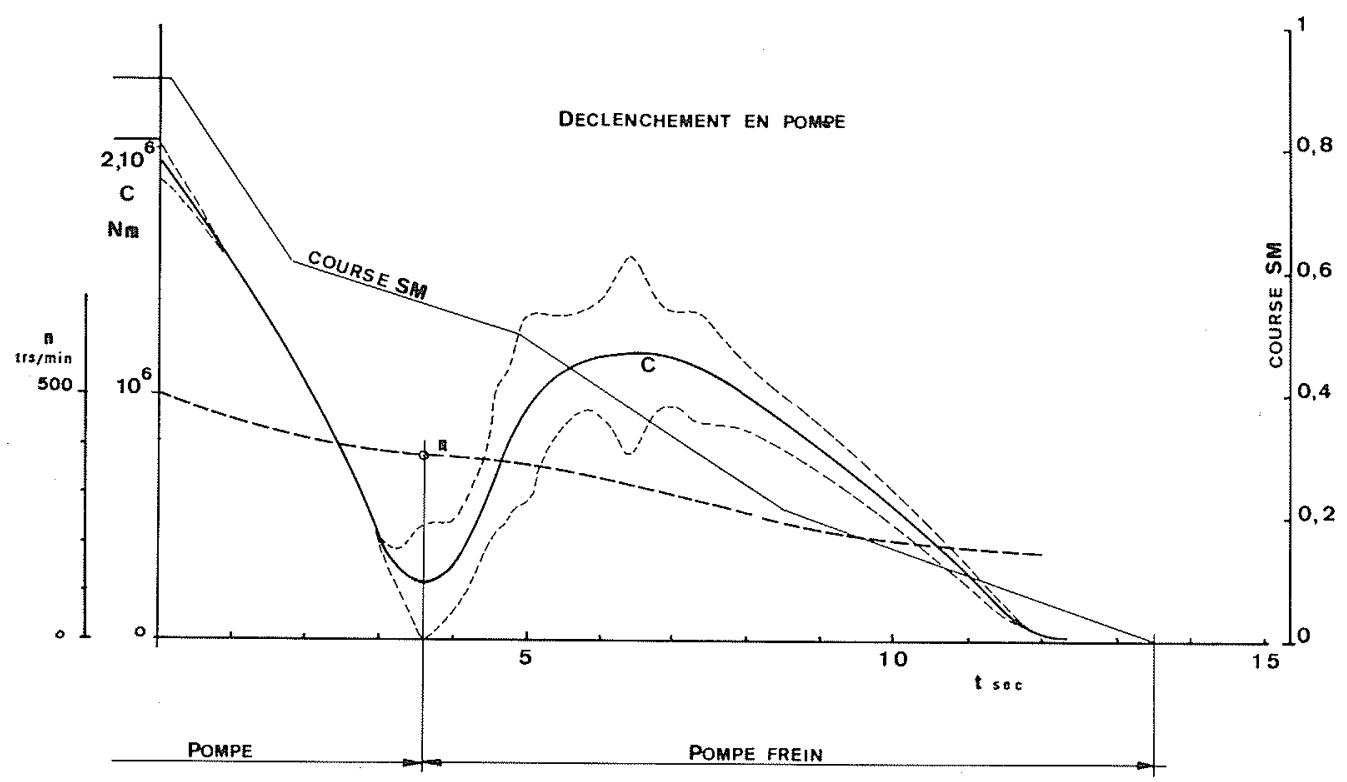

Figure 4 - 
Le graphique $\mathrm{n}^{\circ} 3$ montre l'évolution des paramètres définis ci-dessus au cours de cette opération de démarrage.

On n'observe pas de sollicitations anormales durant le régime transitoire correspondant à l'ouverture du vannage. Seule la pression dans le canal diffuseur côté bâche présente quelques pulsations en début d'ouverture (125 m crête à crête).

\section{Fonctionnement normal en pompe}

En régime normal de pompage, les fluctuations des principaux paramètres mesurés demeurent faibles ainsi que le montre le tableau suivant:

\begin{tabular}{|c|c|c|c|}
\hline Chute brute & $H$ & $371 \mathrm{~m}$ & $\begin{array}{c}\text { Fréquences } \\
\text { caractéristiques }\end{array}$ \\
\hline $\begin{array}{l}\text { Pression bâche } \\
\text { Pression entrefer } \\
\text { Poussée radiale } \\
\text { palier turbine } \\
\text { Couple moteur } \\
\text { Couple sur } \\
\text { tourillon } \\
\text { Poussée axiale }\end{array}$ & $\begin{array}{l}\text { PBa } \\
\text { Pe } \\
\text { P } \\
C \\
C_{T} \\
\text { PAX }\end{array}$ & $\begin{array}{l}403 \pm 7 \mathrm{~m} \\
352 \pm 10 \mathrm{~m} \\
0 \pm 6 \mathrm{~T} \\
197 \pm 0,5 \mathrm{Tm} \\
0,25 \pm \epsilon \mathrm{Tm} \\
55 \pm 4 \mathrm{~T}\end{array}$ & $\begin{array}{l}\text { fo, } 2 \text { fo, } Z_{R} \text { fo } \\
\text { fo } \\
\text { fo, fo } \\
\text { fo, } 2 \text { fo, } Z_{R} \text { fo, } \\
2 Z_{R} \text { fo } \\
\text { fo }\end{array}$ \\
\hline
\end{tabular}

\section{Déclenchement en pompe}

Sur la figure 4, sont tracées les variations de couple moteur, la vitesse de rotation et la course du servomoteur. On remarquera la très faible fluctuation du couple sur l'arbre dans le quadrant pompe.

Celles-ci deviennent importantes au voisinage du débit nul et atteignent leur maximum dans le quadrant pompe frein; on enregistre en effet des amplitudes de $80 \mathrm{Tm}$ crête a crête.

L'analyse spectrale montre que ces fluctuations se produisent à la fréquence de torsion de l'arbre.

La figure 5 montre l'évolution des pressions dans la bâche et l'entrefer, ainsi que du couple sur un tourillon de directrice. Les fluctuations de ce couple son. également maximales dans le quadrant pompe frein et atteignent $3,6 \mathrm{Tm}$ crête à crête. L'analyse spectrale met en évidence la fréquence de passage des aubes de la roue et son premier harmonique.

La figure 6 représente les fluctuations de pression dans la bâche et entrefer pendant le déclenchement. Dans cette phase transitoire, nous retrouvons la fréquence de passage des aubes de la roue.

Sur la figure 7, sont tracés les couples moyens sur les tourillons mesurés sur le prototype et calculés d'après essais sur le modèle réduit. Nous constatons une bonne concordance entre ces deux courbes.

L'évolution des poussées radiales pendant le déclenchement est représenté sur les figures 8 et 9 . On constate une poussée d'environ $25 \mathrm{~T}$ au voisinage du débit nul en direction du bec de bâche, alors qu'en marche normale, la poussée moyenne est pratiquement nulle.

Les fluctuations de la poussée radiale sur le palier turbine atteint un maximum de $\pm 52 \mathrm{~T}$. Ces fluctuations ont une fréquence voisine de la fréquence de rotation.
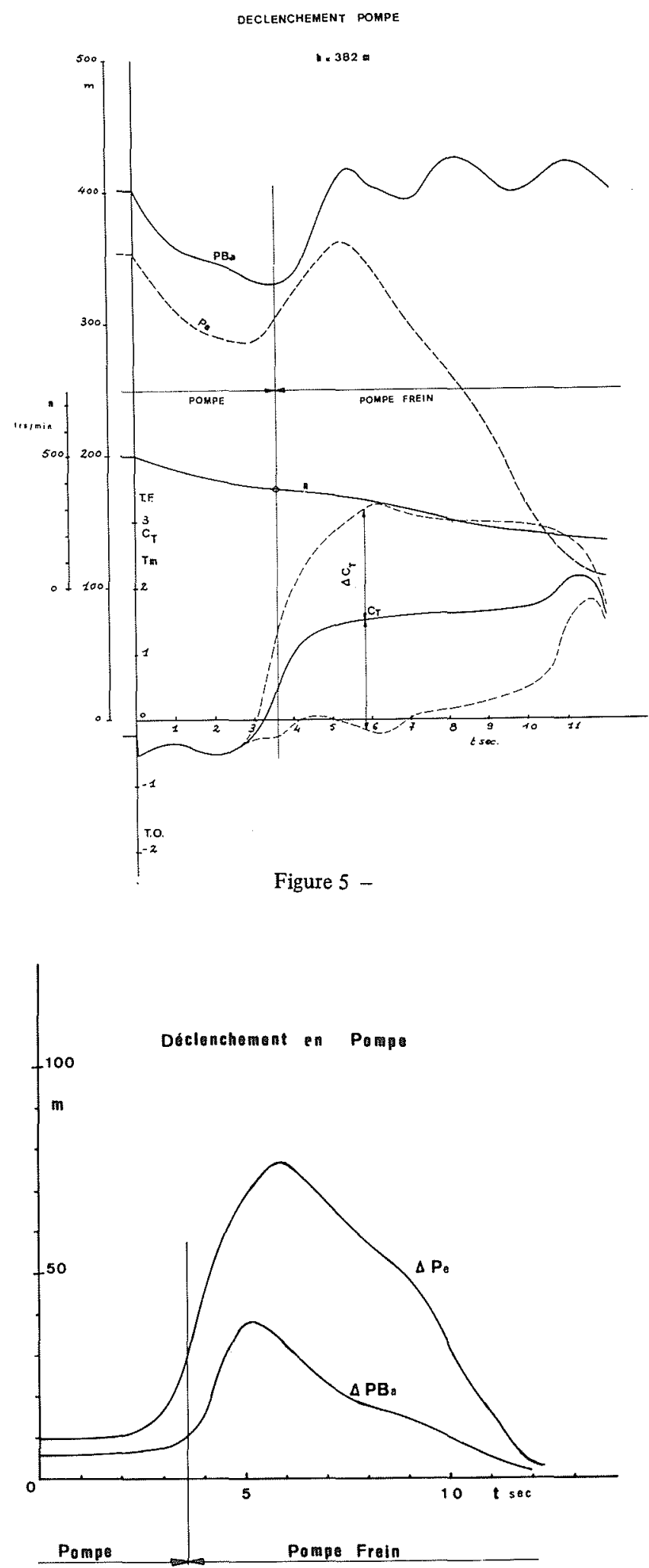

Figure 6 -

Au cours du déclenchement, la poussée axiale décroît et s'inverse dans le quadrant pompe frein où ses fluctuations atteignent $\pm 42 \mathrm{~T}$ à la fréquence de rotation.

La figure 10 se rapporte à un essai de déclenchement avec dévirage. 
DECLENCHEMENT EN POMPE

$h=382 \mathrm{~m}$

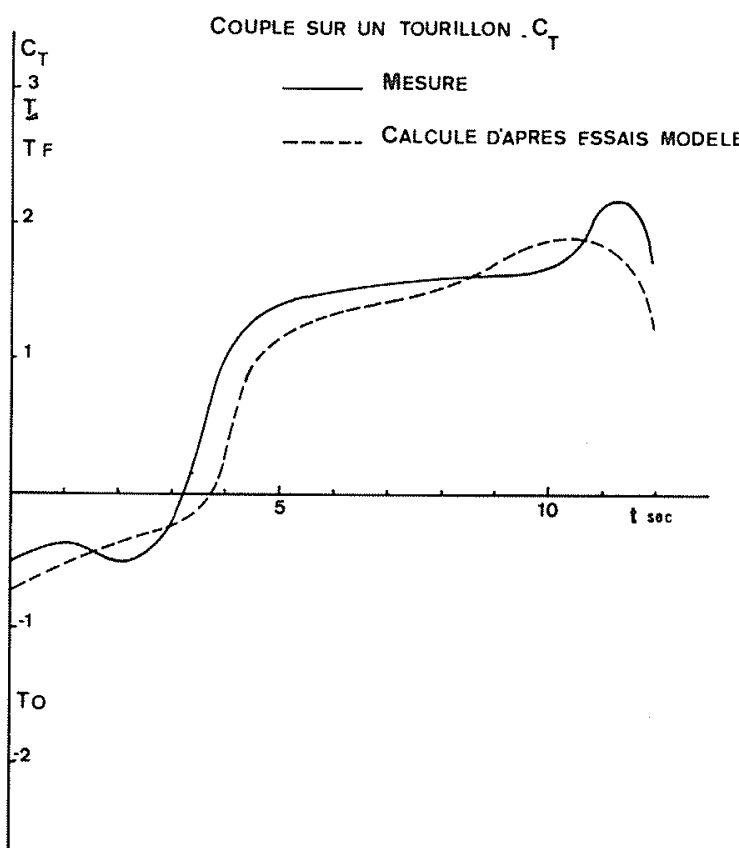

Figure 7 -

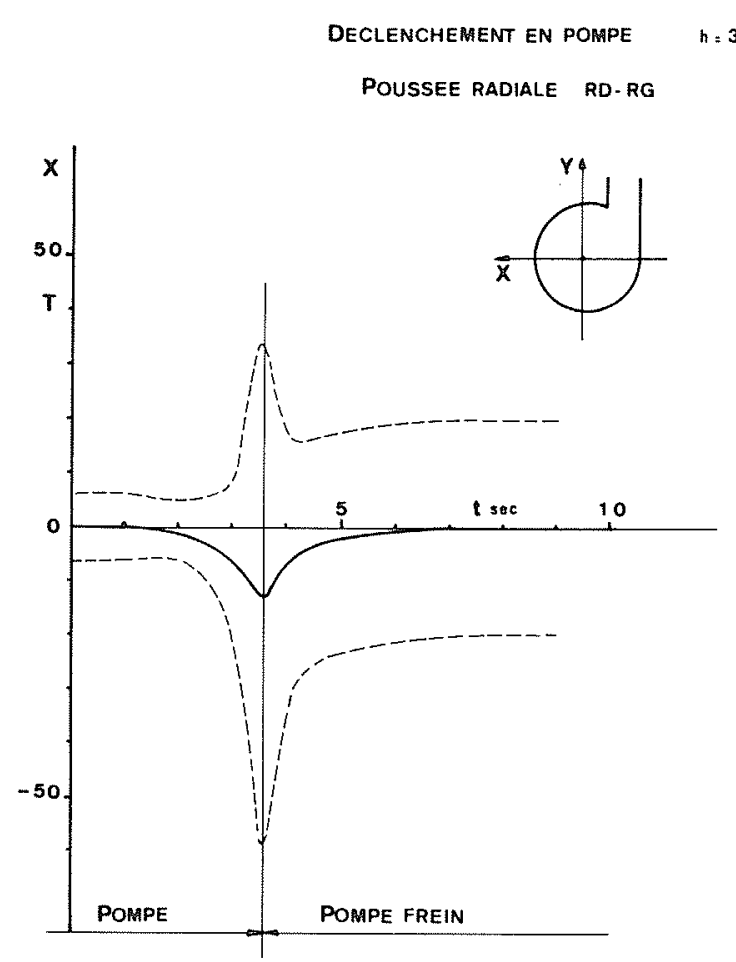

Figure 8 -

\section{Fonctionnement normal en turbine}

Pour ce mode de fonctionnement, nous nous limitons à présenter des résultats relatifs à la poussée radiale, l'analyse du comportement de l'arbre ayant été examiné dans une précédente communication.
Poussee radiale hP. BP

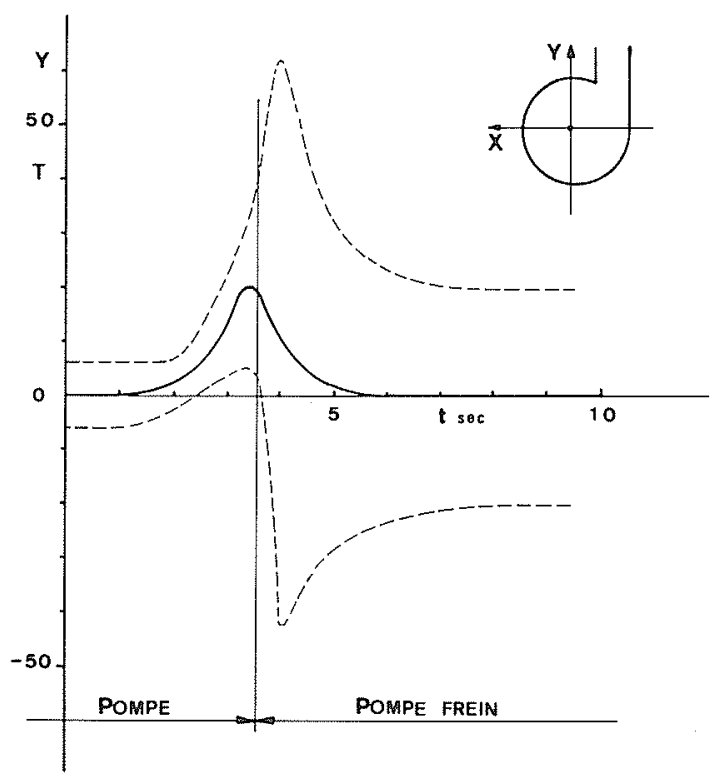

Figure 9 -

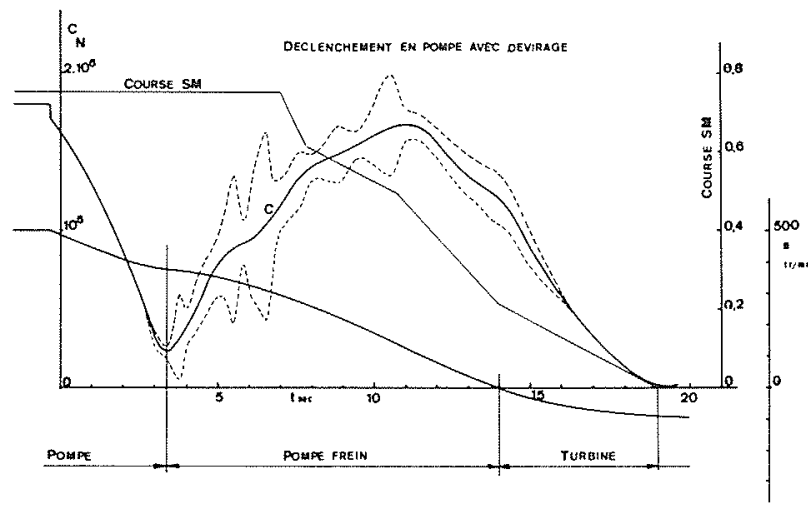

Figure 10 -

La figure 11 représente d'une part des fluctuations de cette poussée en fonction de la charge du groupe, d'autre part une reproduction des enregistrements effectués.

On remarque que ces fluctuations sont maximales en marche à vide et décroissent quand la charge augmente.

L'analyse spectrale des enregistrements met en évidence une force tournante à la fréquence de rotation du groupe.

\section{Déclenchement en turbine}

Sur les figures 12 à 14 , on retrouve l'évolution des paramètres enregistrés en pompe.

Pressions bâche et entrefer - Vitesse-couple sur l'arbre et sur un tourillon de directrice.

On constate que les fluctuations des pressions et des couples sont maximales au voisinage de l'emballement et dans la zone de dissipation d'énergie. 

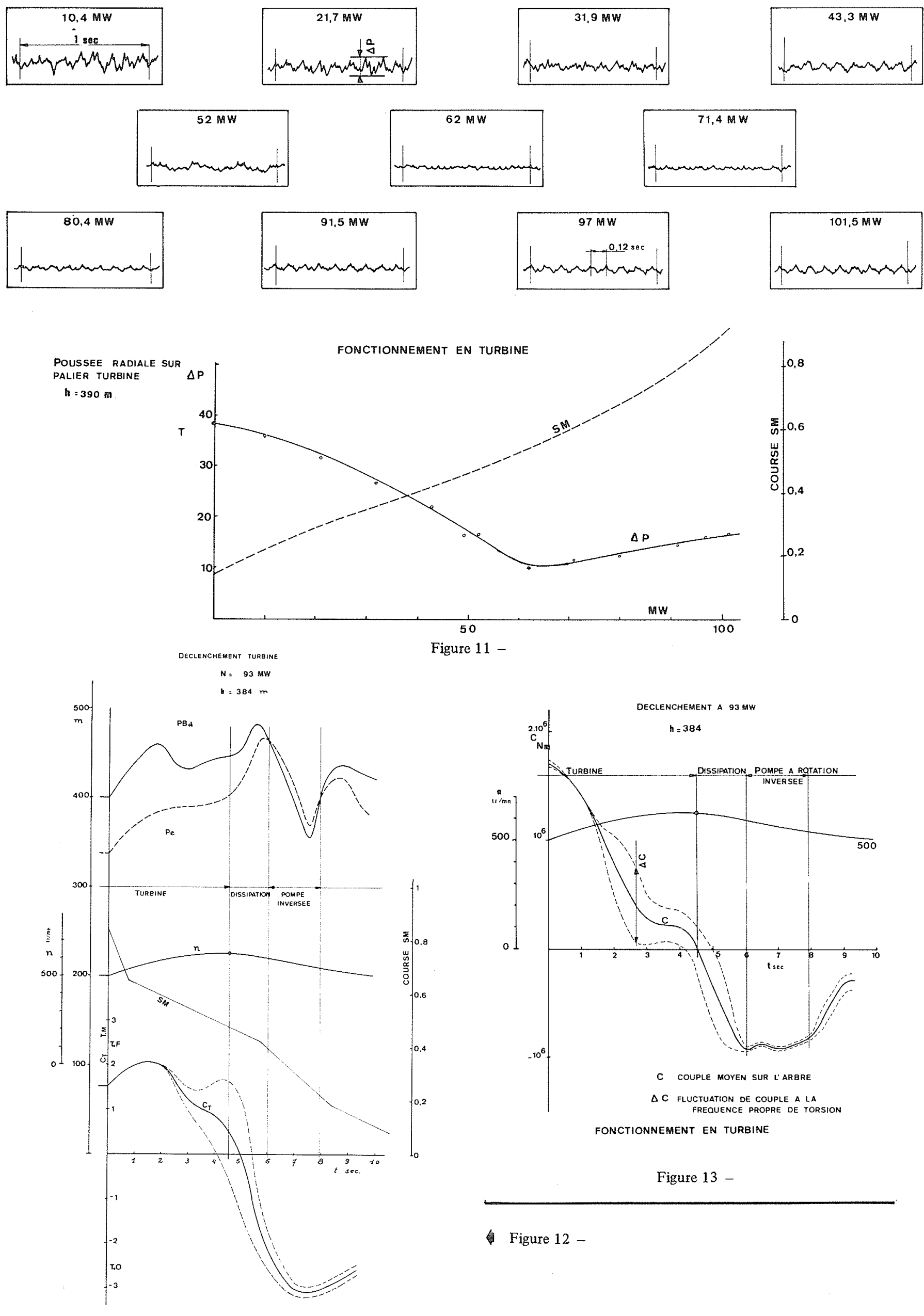

FONCTIONNEMENT EN TURBINE

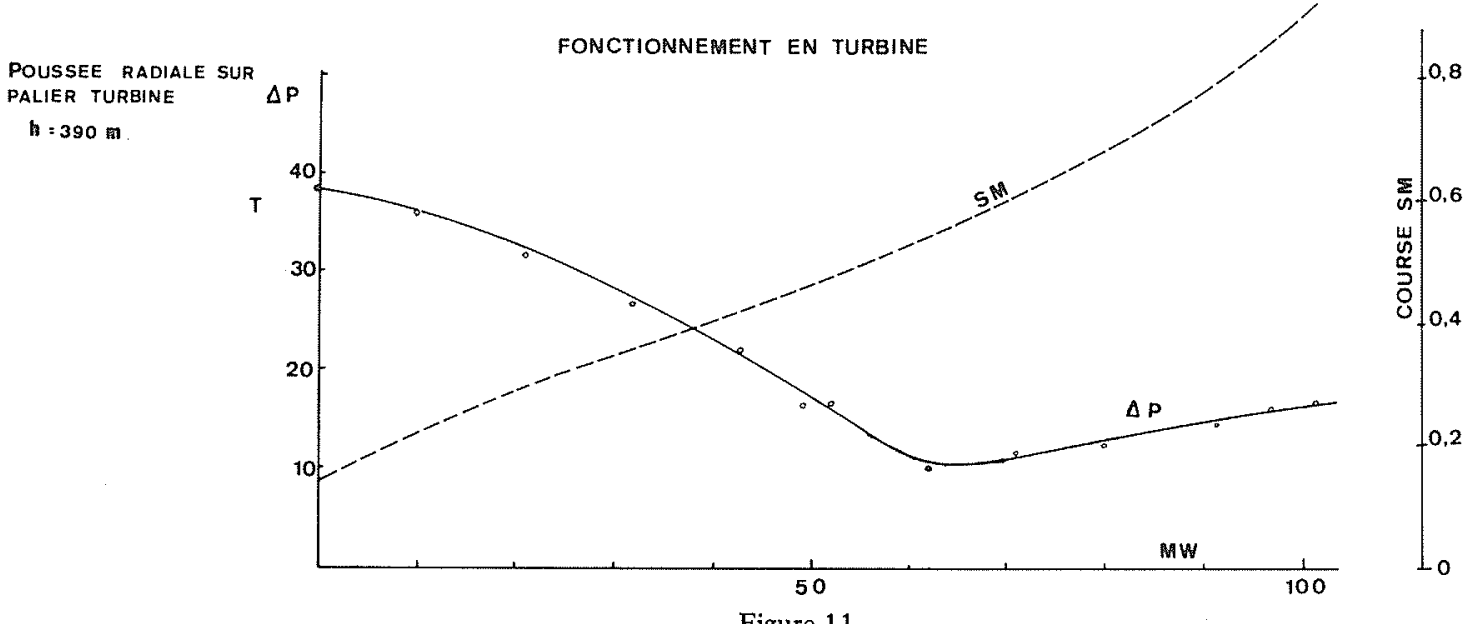

Figure 11 -

100

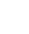

Figure 13 -

\section{Figure 12 -}




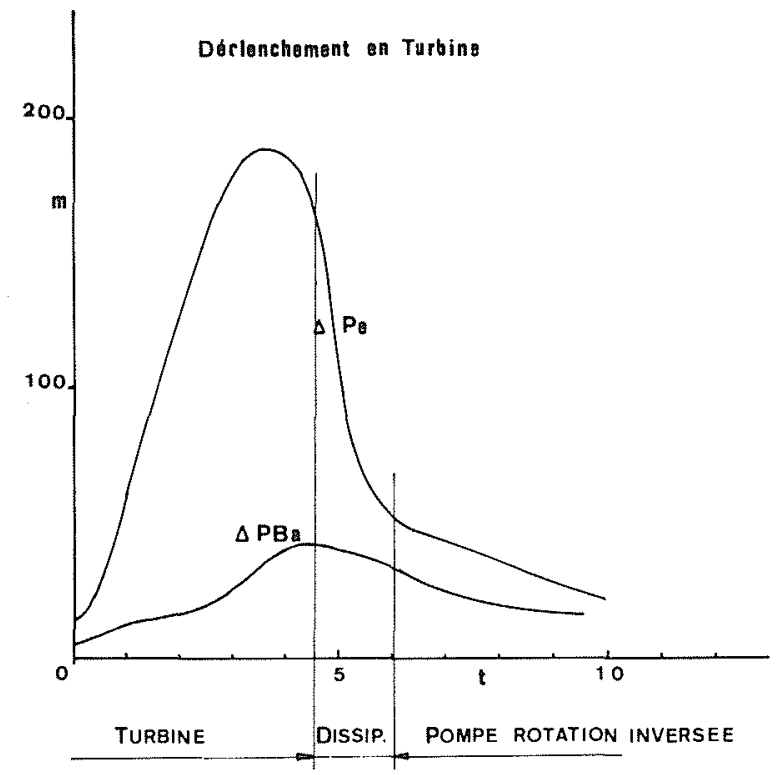

Figure 14 -

Par contre, dans le quadrant pompe à rotation inversée, les fluctuations sont très faibles.

Les pressions dans l'entrefer fluctuent principalement à la fréquence de passage des aubes. On note également une composante plus faible à la fréquence de rotation. On observe le même phénomène en ce qui concerne le couple sur le tourillon de directrice.

Comme pour le fonctionnement en pompe, on observe sur la figure 15 une bonne concordance entre le modèle et le prototype dans l'évolution du couple moyen sur une directrice.

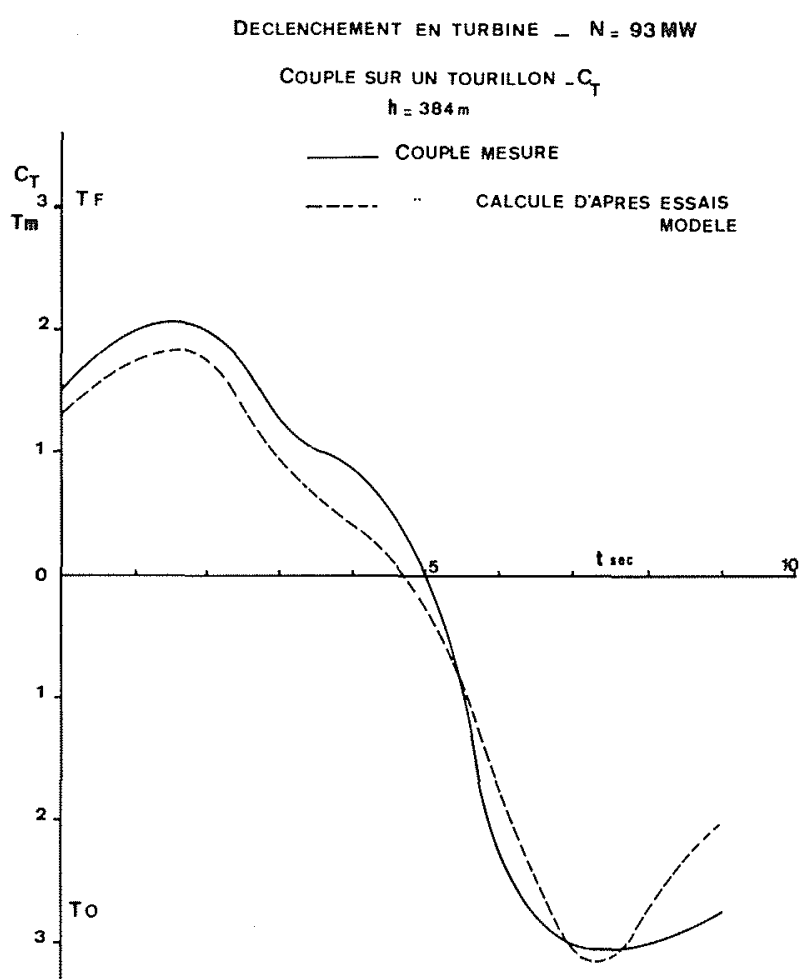

Figure 15 -
DECLENCHEMENT TURBINE

$h=384 m$ POUSSEE RADIALE/PaLIER T

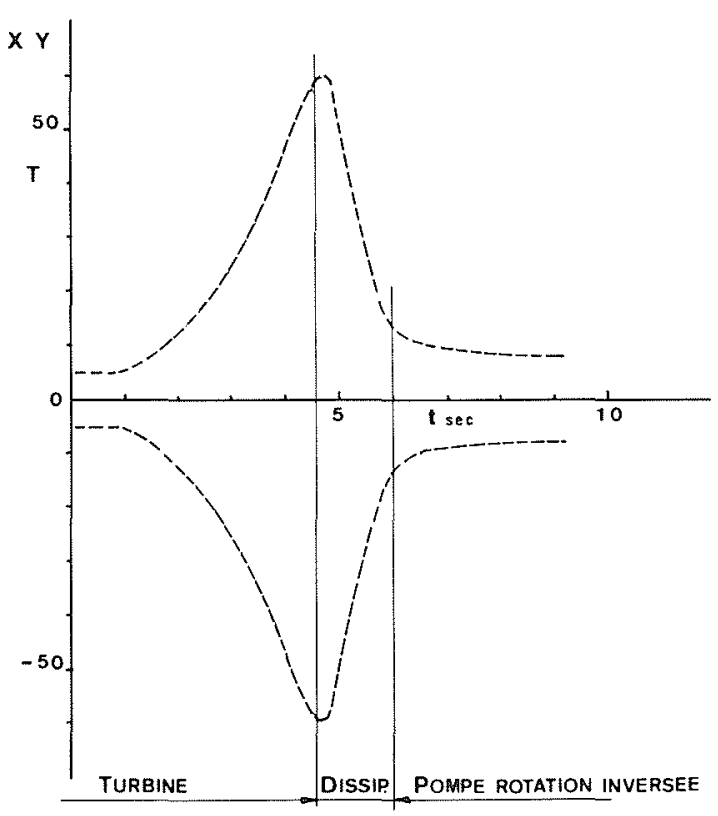

Figure 16 -

En ce qui conceme l'évolution de la poussée radiale, on observe des fluctuations importantes au voisinage de l'emballement, mais la valeur moyenne demeure pratiquement nulle (fig. 16). L'évolution des fréquences a été analysée dans un article précédent.

Enfin, la poussée axiale accuse également une forte augmentation au voisinage de l'emballement - de $70 \mathrm{~T}$ en marche normale elle passe à $240 \mathrm{~T}$ avec des fluctuations de $60 \mathrm{~T}$ crête à crête la fréquence dominante étant la fréquence de rotation.

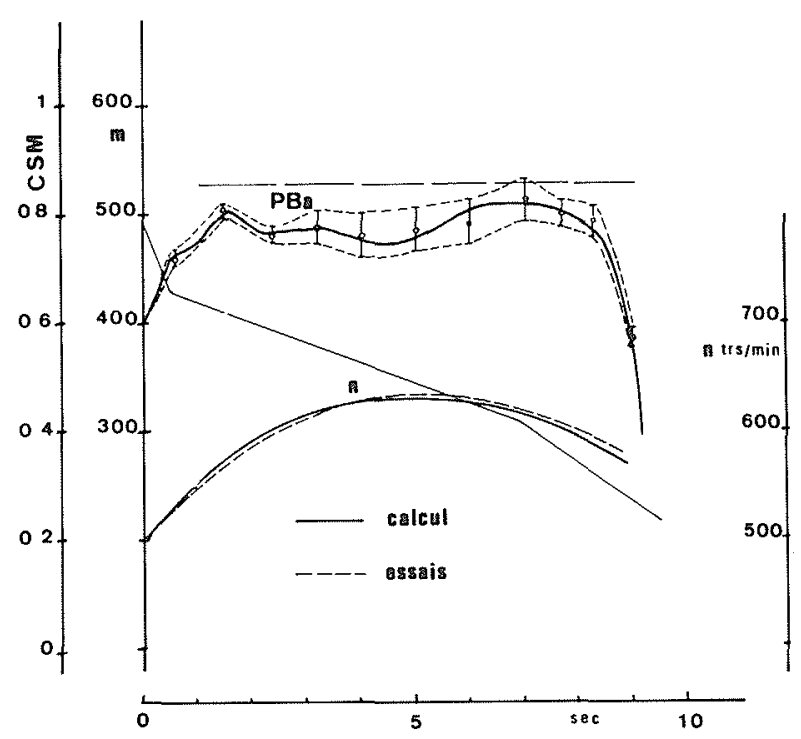

Décleneherant da 4 Turbinas

$h=390 \mathrm{~m}$

Figure 17 - 
Déclenchements - Loi de fermeture du distributeur

La détermination de la loi de mancuvre a obéi aux divers impératifs suivants :

- loi unique en pompe et en turbine pour des raisons de sécurité

- respect des surpressions et limitation des survitesses aux valeurs les plus faibles possibles. Nous avons vu en effet que les sollicitations augmentent avec la survitesse

- nécessité contractuelle d'éviter le dévirage en pompe Ces contraintes parfois contradictoires nous ont conduits à utiliser une loi complexe à 4 pentes.

La mise au point de cette loi a nécessité une mise au point sur le site en combinant essais et calculs. $\mathrm{La}$ figure 17 représente la comparaison des sur- pressions et survitesses calculées et mesurées consécutives à un déclenchement de 4 groupes en turbine avec la loi de fermeture définitive.

Les résultats de déclenchement en pompe ont donné des concordances identiques entre calculs et mesures.

\section{Conclusion}

Les résultats d'essais confirment la validité des hypothèses et méthodes de calcul utilisées pour le dimensionnement de ces machines et pour l'étude des phénomènes transitoires.

Ils permettent d'envisager avec confiance une nouvelle étape sur des puissances unitaires plus importantes et des chutes plus élevées. 